\title{
HIGHER ORDER TRACE RELATIONS FOR SCHRÖDINGER OPERATORS
}

\author{
F. Gesztesy ${ }^{1}$, H. Holden 2 , B. Simon ${ }^{3}$, And Z. ZhaO ${ }^{1}$ \\ Dedicated to Henry P. McKean on the occasion of his sixtieth birthday
}

\begin{abstract}
We extend the trace formula recently proven for general one-dimensional Schrödinger operators which obtains the potential $V(x)$ from a function $\xi(x, \lambda)$ by deriving trace relations computing moments of $\xi(x, \lambda) d \lambda$ in terms of polynomials in the derivatives of $V$ at $x$. We describe the relation of those polynomials to $\mathrm{KdV}$ invariants. We also discuss trace formulae for analogs of $\xi$ associated with boundary conditions other than the Dirichlet boundary condition underlying $\xi$.
\end{abstract}

\section{$\S 1$. Introduction}

This paper is one in a series [14-20] concerning a basic function, $\xi(\lambda, x)$, associated to any one-dimensional Schrödinger operator, $H=-\frac{d^{2}}{d x^{2}}+V$ in $L^{2}(\mathrm{R})$ and its application to inverse spectral problems. A basic formula proven in [18] is that

$$
V(x)=E_{0}+\lim _{\alpha \downarrow 0} \int_{E_{0}}^{\infty} d \lambda e^{-\alpha \lambda}(1-2 \xi(\lambda, x)),
$$

where $E_{o}=\inf \operatorname{spec}\left(-\frac{d^{2}}{d x^{2}}+V\right)$. (1.1) was proven in [18] assuming $V$ is bounded below, continuous, and $|V(x)| \leq C_{1} e^{C_{2} x^{2}}$.

Our definition of $\xi$ is

$$
\xi(\lambda, x):=\frac{1}{\pi} \operatorname{Arg}((G(\lambda+i 0, x, x))
$$

(where $G\left(z, x, x^{\prime}\right)$ denotes the Green's function of $H$, that is, the integral kernel of ( $H-$ $z)^{-1}$ ), although we derived that from a basic definition as the Krein spectral shift in going

\footnotetext{
${ }^{1}$ Department of Mathematics, University of Missouri, Columbia, MO 65211. E-mail for F.G.: mathfg @mizzou1.missouri.edu; e-mail for Z.Z.: mathzz@mizzou1.missouri.edu

2 Department of Mathematical Sciences, The Norwegian Institute of Technology, University of Trondheim, N-7034 Trondheim, Norway. E-mail: holden@imf.unit.no

3 Division of Physics, Mathematics, and Astronomy, California Institute of Technology, 253-37, Pasadena, CA 91125. This material is based upon work supported by the National Science Foundation under Grant No. DMS-9101715. The Government has certain rights in this material.

To be submitted to Commun. Pure Appl. Math.
} 
from $H$ to $H_{D ; x}$, the operator on $L^{2}(-\infty, x) \oplus L^{2}(x, \infty)$ with Dirichlet boundary condition at $x$.

The key to (1.1) then was

$$
\operatorname{Tr}\left(e^{-t H}-e^{-t H_{D ; x}}\right)=\frac{1}{2}(1-t V(x)+o(t)) \quad \text { as } t \downarrow 0 .
$$

(1.3) is related to (1.1) because the Krein spectral shift [30] is a function $0 \leq \xi(\lambda, x) \leq 1$ obeying

$$
\operatorname{Tr}\left[f(H)-f\left(H_{D ; x}\right)\right]=-\int_{E_{o}}^{\infty} d \lambda f^{\prime}(\lambda) \xi(\lambda, x)
$$

for a rich set of $f$ 's including exponentials (e.g., $f \in C^{2}(\mathrm{R}),\left(1+\lambda^{2}\right) f^{(j)} \in L^{2}((0, \infty))$, $j=1,2$ and also $\left.f(\lambda)=(\lambda-z)^{-1}, z \in \mathrm{C} \backslash\left[E_{o}, \infty\right)\right)$ so that

$$
\operatorname{Tr}\left(e^{-t H}-e^{-t H_{D ; x}}\right)=t \int_{E_{o}}^{\infty} d \lambda e^{-t \lambda} \xi(\lambda, x) .
$$

One of our goals in the present paper is to prove (1.1) in greater generality; we only need $V$ bounded from below with no growth restriction at infinity. $V$ need not be continuous; a local $L^{1}$ condition suffices. (1.1) then holds at points of Lebesgue continuity of $V$.

Our main goal though is to prove higher order trace formulas. In great generality (suppose $V$ has an asymptotic Taylor series at $x_{0}$ ), we'll extend (1.3) to

$$
\operatorname{Tr}\left(e^{-t H}-e^{-t H_{D ; x_{0}}}\right) \underset{t \downarrow 0}{\sim}-\sum_{j=0}^{\infty} s_{j}\left(x_{0}\right) t^{j},
$$

where $s_{j}(x)=(-1)^{j+1}(j !)^{-1} r_{j}(x)$ and the $r_{j}$ are $\mathrm{KdV}$ invariants defined recursively in Theorem 5.1 below. With more information one can relate this to a similar formula in terms of $\xi$ (for simplicity of notation we suppose that $E_{o}=0$ ):

$$
r_{j}\left(x_{0}\right)=j \lim _{\alpha \downarrow 0} \int_{0}^{\infty} d \lambda e^{-\lambda \alpha} \lambda^{j-1}\left(\frac{1}{2}-\xi\left(\lambda, x_{0}\right)\right) .
$$

The key to handling potentials with no growth condition at infinity is a path space representation for $\operatorname{Tr}\left(e^{-t H}-e^{-t H_{D ; x}}\right)$. Properties of the paths needed are proven in $\S 2$. Then in $\S 3$, we prove (1.1) for general $V$. In $\S 4$, we show that $\operatorname{Tr}\left(e^{-t H}-e^{-t H_{D ; x}}\right)$ has an asymptotic expansion to all orders in $t$ at $t=0$ if $V$ is $C^{\infty}$. In $\S 5$, we relate the coefficients of this expansion to the $\mathrm{KdV}$ invariants, and in $\S 6$ we discuss what happens if boundary conditions other than Dirichlet are used.

Historically, trace formulas for Schrödinger operators on a finite interval originated with a 1953 paper by Gel'fand and Levitan [11] with later contributions by Dikii [6], Gel'fand 
[9], Halberg-Kramer [22], and Gilbert-Kramer [21]. The case of periodic potentials was first studied by Hochstadt [25] who obtained a trace formula for $V(x)-V(0)$ in terms of appropriate Dirichlet eigenvalues in the special case of finite-gap potentials. The periodic trace formula (5.59) for finite-gap potentials $V(x)$ in terms of Dirichlet eigenvalues was first derived by Dubrovin [7]. The periodic trace formulas (5.59) for all higher order Kortewegde Vries invariants $s_{j}(x)$ were first proven in 1975 by McKean-van Moerbeke [35] and independently by Flaschka [8], the trace formula for $s_{1}(x)=\frac{1}{2} V(x)$ for general periodic $C^{3}$ potentials by Trubowitz [40] in 1977. More recently, the trace formula (5.59) for $V(x)$ has been extended to certain classes of almost periodic potentials in Levitan [32,33], Kotani-Krishna [29], and Craig [2]. Analogous trace formulas for Schrödinger operators on the real line with potentials decaying sufficiently rapidly at infinity have been studied in 1979 by Deift and Trubowitz [5], and more recently by Venakides [41], Gesztesy-Holden [13], Gesztesy [12], and Gesztesy-Holden-Simon-Zhao [14].

These trace formulas are a key element of the solution of the inverse spectral problem for periodic potentials and the inverse scattering problem for potentials decaying sufficiently fast at infinity (see, e.g., [5], [7], [8], [25], [26], [32-36], [40], [41] and the references therein.)

\section{$\S 2$. The Xi Process}

In [18], we introduced a probability measure on the set of paths on $[0,1]$ as follows. Let $\alpha$ be the Brownian bridge, that is, the Gaussian process of $\{\alpha(s)\}_{0 \leq s \leq 1}$ of mean zero and covariance $E_{\alpha}(\alpha(s) \alpha(t))=s(1-t)$ if $s \leq t$. In terms of Brownian motion, one can realize $\alpha$ as $\alpha(s)=b(s)-s b(1)$ (see [37] for discussion of Brownian motion, Gaussian processes, and the Brownian bridge). There is a Baire measure $\mathcal{D} \alpha$ on $C([0,1])$ induced by the process.

Let $d \kappa$ be the measure on $\mathrm{R} \times C([0,1])$ given by $d x \otimes(4 \pi)^{-1 / 2} \mathcal{D} \alpha$ where $d x$ is Lebesgue measure. Let $\omega(s)=x+\alpha(s)$ and let $\Omega_{0} \subset \mathrm{R} \times C([0,1])$ be the set of paths given by $\{\omega \mid \omega(s)=0$ for some $s \in[0,1]\}$. We claim that

$$
\int_{\Omega_{0}} d \kappa=\frac{1}{2}
$$

for the free Feynman-Kac formula says

$$
\begin{aligned}
e^{\Delta / 2}(x, x) & =(4 \pi)^{-1 / 2} \int_{\{\omega(0)=x\}} \mathcal{D} \alpha=(4 \pi)^{-1 / 2}, \\
e^{\Delta_{D} / 2}(x, x) & =(4 \pi)^{-1 / 2} \int_{\{\omega(0)=x ; \omega(s) \neq 0 \text { all } s \in[0,1]\}} \mathcal{D} \alpha,
\end{aligned}
$$


where $\Delta_{D}=\Delta_{D ; 0}$ has a Dirichlet boundary condition at $x=0$. Thus

$$
\begin{aligned}
\int_{\Omega_{0}} d \kappa & =\int_{\mathrm{R}} d x\left[\exp \left(\frac{1}{2} \Delta\right)(x, x)-\exp \left(\frac{1}{2} \Delta_{D}\right)(x, x)\right] \\
& =\int_{\mathrm{R}} d x \exp \left(\frac{1}{2} \Delta\right)(x,-x) \\
& =\int_{\mathrm{R}} d x \exp \left(\frac{1}{2} \Delta\right)(2 x, 0) \\
& =\frac{1}{2} \int_{\mathrm{R}} d y \exp \left(\frac{1}{2} \Delta\right)(y, 0)=\frac{1}{2} .
\end{aligned}
$$

We define the xi process by placing the measure $\mathcal{D} \omega \equiv 2 \chi_{\Omega_{0}} d \kappa$ on $C([0,1])$ with $\omega(s)=$ $x+\alpha(s)$.

The reason for the interest in $\mathcal{D} \omega$ is that by writing (2.3) with a potential, one finds (see [37]):

Proposition 2.1. Let $V$ be bounded below and continuous on $(-\infty, \infty), H=-\frac{d^{2}}{d x^{2}}+V$ on $L^{2}(-\infty, \infty)$ and let $H_{D}=-\frac{d^{2}}{d x^{2}}+V$ on $L^{2}(-\infty, 0) \oplus L^{2}(0, \infty)$ with a Dirichlet boundary condition at $x=0$ (i.e., $H_{D}=H_{D ; 0}$ ). Then

$$
\operatorname{Tr}\left(e^{-t H}-e^{-t H_{D}}\right)=\frac{1}{2} E_{\omega}\left(\exp \left(-t \int_{0}^{1} d s V(\sqrt{2 t} \omega(s))\right)\right) .
$$

The Feynman-Kac formula (2.4) will be critical for the proof of our higher order trace relations. We'll need the following technical result (we use the notation employed in [37], i.e., $E(f)=\int_{\Omega} f d \mu, E(A)=\int_{A} d \mu=\mu(A), E(f ; A)=\int_{A} f d \mu$, etc., where $(\Omega, \mathcal{F}, \mu)$ denotes a probability space, $A \in \mathcal{F}, f: \Omega \rightarrow \mathrm{R}$ is $\mathcal{F}$-measurable):

Theorem 2.2. $E_{\omega}\left(\left\{\omega\left|\sup _{0 \leq s \leq 1}\right| \omega(s) \mid \geq a\right\}\right) \leq C_{1} \exp \left(-C_{2} a^{2}\right)$ for some $C_{1}, C_{2}>0$.

Proof. Look at sets on $\mathrm{R} \times C([0,1])$ with measure $d \kappa$. Let $T_{a}=\left\{\omega \in \Omega_{0}\left|\sup _{0 \leq s \leq 1}\right| \omega(s) \mid \geq a\right\}$. Then

$$
\begin{aligned}
& T_{a} \subset\left\{\omega \in \Omega_{0}|| \omega(0) \mid>\frac{a}{2}\right\} \cup\left\{\omega|| \omega(0) \mid<\frac{a}{2}, \sup _{0 \leq s \leq 1} \omega(s) \geq a\right\} \\
& \cup\left\{\omega|| \omega(0) \mid<\frac{a}{2}, \inf _{0 \leq s \leq 1} \omega(s) \leq-a\right\} \\
& \equiv T_{a}^{(1)} \cup T_{a}^{(2)} \cup T_{a}^{(3)} .
\end{aligned}
$$


Notice that we have dropped the $\omega \in \Omega_{0}$ condition from $T_{a}^{(i)}, i=2,3$. In each case, we have a single condition on a value that we must take, for example:

$$
T_{a}^{(2)}=\left\{\omega|| \omega(0) \mid<\frac{a}{2}, \omega(s)=a \text { for some } s \in[0,1]\right\} .
$$

Thus, each $\int_{T_{a}^{(i)}} d x$ can be written in terms of a Dirichlet boundary condition (at $0, a,-a$, respectively) and then by the method of images in terms of the free heat kernel of $e^{\Delta / 2}$. Explicitly,

$$
\begin{aligned}
\int_{T_{a}^{(1)}} d x & =\int_{|x|>a / 2} d x e^{\Delta / 2}(x,-x), \\
\int_{T_{a}^{(2)}} d x & =\int_{|x|<a / 2} d x e^{\Delta / 2}(x, 2 a-x), \\
\int_{T_{a}^{(3)}} d x & =\int_{|x|<a / 2} d x e^{\Delta / 2}(x,-2 a-x)
\end{aligned}
$$

and each of these is bounded by $C_{1} \exp \left(-C_{2} a^{2}\right)$.

Remark 2.3. The xi process, $\omega$, is not Gaussian. However, the process, L, obtained by reflecting $\omega$ in the first time it hits 0 is Gaussian. It will be discussed in [16] where an alternate proof of Theorem 2.2 can be found.

\section{$\S 3$. Zeroth Order Asymptotics}

Here we'll prove the following generalization of a result we proved in [18].

Theorem 3.1. Let $V$ be a measurable function of $\mathrm{R}$ obeying

(i) $\sup _{n \in \mathrm{N}} \int_{n}^{n+1}\left|V_{-}(x)\right| d x<\infty$,
(ii) $\int_{n}^{n+1}\left|V_{+}(x)\right| d x<\infty$ for all $n \in \mathrm{N}$,

where $V_{ \pm}(x)=\max _{\min }(V(x), 0)$. Let $H=-\frac{d^{2}}{d x^{2}}+V$ on $L^{2}(-\infty, \infty)$ and $H_{D ; y}=-\frac{d^{2}}{d x^{2}}+V$ on $L^{2}(-\infty, y) \oplus L^{2}(y, \infty)$ with Dirichlet boundary conditions at $y$. Let $\xi(\lambda, y)$ be the Krein spectral shift for $H$ to $H_{D ; y}$. Let $E_{o}=\inf \operatorname{spec}(H)$. If $x$ is a point of Lebesgue continuity for $V$, then

$$
V(x)=E_{o}+\lim _{\alpha \downarrow 0} \int_{E_{o}}^{\infty} d \lambda e^{-\alpha \lambda}[1-2 \xi(\lambda, x)] .
$$

Remark 3.2. This generalizes the result in [18] in three ways. There we assumed $V(x) \leq$ $C_{1} e^{C_{2} x^{2}}$, we supposed $V$ bounded below and that $V$ is continuous. 
Proof of Theorem 3.1. For notational simplicity, suppose $x=0, E_{o}=0$, and write $H_{D ; 0}=$ $H_{D}$. Let $W(y)=V(y)$ if $|y| \leq 1$ and $W(y)=0$ if $|y| \geq 1$. Then, by (2.4)

$$
\begin{aligned}
\operatorname{Tr}\left(e^{-t H}-e^{-t H_{D}}\right)= & \frac{1}{2} E_{\omega}\left(\exp \left(-t \int_{0}^{1} d s V(\sqrt{2 t} \omega(s))\right)\right) \\
= & \frac{1}{2} E_{\omega}\left(\chi\left(\sup _{0 \leq s \leq 1}(\sqrt{2 t}|\omega(s)|<1)\right) \exp \left(-t \int_{0}^{1} d s V(\sqrt{2 t} \omega(s))\right)\right) \\
& +O\left(C_{1} e^{-C_{2} / t}\right) \\
= & \frac{1}{2} E_{\omega}\left(\chi\left(\sup _{0 \leq s \leq 1}(\sqrt{2 t}|\omega(s)|<1)\right) \exp \left(-t \int_{0}^{1} d s W(\sqrt{2 t} \omega(s))\right)\right) \\
\quad+O\left(C_{1} e^{-C_{2} / t}\right) & \frac{1}{2} E_{\omega}\left(\exp \left(-t \int_{0}^{1} d s W(\sqrt{2 t} \omega(s))\right)\right)+O\left(C_{1} e^{-C_{2} / t}\right)
\end{aligned}
$$

where (3.1) follows from Theorem 2.2, the Schwartz inequality and the estimate that $\operatorname{Tr}\left(e^{-t \tilde{H}}-e^{-t \tilde{H}_{D}}\right)<\infty$ with $\tilde{H}=-\frac{d^{2}}{d x^{2}}+2 V$.

The general proof when only (ii) holds is a little complicated so we consider first the case where $V$ is bounded. Then since $\left|e^{x}-1-x\right| \leq \frac{1}{2} x^{2} e^{|x|}$, we have by (3.2) that

$$
\begin{aligned}
\operatorname{Tr}\left(e^{-t H}-e^{-t H_{D}}\right) & =\frac{1}{2}\left(1-t E_{\omega}\left(\int_{0}^{1} d s W(\sqrt{2 t} \omega(s))\right)\right)+O\left(t^{2}\right)+O\left(C_{1} e^{-C_{2} / t}\right) \\
& =\frac{1}{2}(1-t a)+o(t),
\end{aligned}
$$

where

$$
a=\lim _{t \downarrow 0} \int_{\mathrm{R}} d x V(x) g_{t}(x)
$$

with $g_{t}(x)$ the probability distribution for $\sqrt{2 t} \omega(s)$ with $s$ distributed uniformly in $[0,1]$. Then $g_{t}(x)=\frac{1}{\sqrt{2 t}} g_{1}(x / \sqrt{2 t})$ so by general principles, $a=V(0)$ since $x=0$ is a point of Lebesgue continuity for $V$.

Next note that

$$
\operatorname{Tr}\left(e^{-t H}-e^{-t H_{D}}\right)=t \int_{0}^{\infty} d \lambda e^{-t \lambda} \xi(\lambda, 0), \quad 1=t \int_{0}^{\infty} e^{-t \lambda} d \lambda
$$


Thus

$$
\begin{aligned}
\lim _{t \downarrow 0} \int_{0}^{\infty} d \lambda e^{-t \lambda}(1-2 \xi(\lambda, 0)) & =-\lim _{t \downarrow 0} 2\left[\operatorname{Tr}\left(e^{-t H}-e^{-t H_{D}}\right)-\frac{1}{2}\right] / t \\
& =a=V(0)
\end{aligned}
$$

by (3.3).

Turning to the general case, as above we can suppose that $V$ is supported on $[-1,1]$ (i.e., is equal to $W$ ), and we need only prove (3.3) assuming $V \in L^{1}(\mathrm{R})$. Let $P(x, y ; t, \mu)$ be the integral kernel of $\exp \left(-t\left(-\frac{d^{2}}{d x^{2}}+\mu V\right)\right), P_{t}(x, y) \equiv P(x, y, t, \mu=1), P_{t}^{(o)}(x, y)=$ $P(x, y, t, \mu=0)=(4 \pi t)^{-1 / 2} \exp \left(-(x-y)^{2} / 4 t\right)$. By the method of images:

$$
\operatorname{Tr}\left(e^{-t H}-e^{-t H_{D}}\right)=\int_{\mathrm{R}} d x P_{t}(x,-x) .
$$

Moreover, (see, e.g., [38]) uniformly on $\mu, t \in[0,1]$ :

$$
P(x, y, t, \mu) \leq C_{\epsilon} t^{-1 / 2} \exp \left(-(x-y)^{2} /(4+\epsilon)\right) .
$$

By (3.5) for any $\alpha>0$, we can integrate in (3.4) over $|x|<t^{1 / 2-\alpha}$ with an error $O\left(e^{-d / t^{2 \alpha}}\right)$.

By DuHamel's formula:

$$
\frac{d}{d \mu} P(x, y, t, \mu)=-\int_{0}^{t} d s d z P(x, z, s, \mu) V(z) P(z, y, t-s, \mu) .
$$

Thus, iterating and using (3.5) in the form $|P(x, y ; t, \mu)| \leq C_{\epsilon} t^{-1 / 2}$,

$$
\begin{aligned}
\left|\frac{d^{k}}{d \mu^{k}} P(x, y, t, \mu)\right| & \leq\left(\int_{[-1,1]} d z|V(z)|\right)^{k} C_{k} \int_{\substack { s_{i} \geq 0 \\
\begin{subarray}{c}{k \\
\sum_{i=1}^{k} s_{i}<t{ s _ { i } \geq 0 \\
\begin{subarray} { c } { k \\
\sum _ { i = 1 } ^ { k } s _ { i } < t } }\end{subarray}} \prod_{j} d s_{j}\left[\prod_{i=1}^{k} s_{i}^{-1 / 2}\right]\left(t-\sum_{\ell=1}^{k} s_{\ell}\right)^{-1 / 2} \\
& \leq \tilde{C}_{k} t^{(k-1) / 2} .
\end{aligned}
$$

Thus by Taylor's theorem with remainder if we take the $0,1,2$ terms in the Taylor expansion about $\mu=0$, the error in

$$
\int_{|x|<t^{1 / 2-\alpha}} d x P_{t}(x,-x)
$$


is bounded by $C t t^{1 / 2-\alpha}=o(t)$. Thus up to $o(t)$ errors $\operatorname{Tr}\left(e^{-t H}-e^{-t H_{D}}\right)=\alpha+\beta+\gamma$, where

$$
\begin{aligned}
& \alpha=\int_{\mathrm{R}} d x P_{t}^{(o)}(x,-x), \\
& \beta=-\int_{0<s<t} d z d s d x P_{s}^{(o)}(x, z) V(z) P_{t-s}^{(o)}(z,-x), \\
& \gamma=\frac{1}{2} \int_{\substack{0<u<t \\
0<s<t \\
u+s<t}} d u d s d x d z d w P_{u}^{(o)}(x, z) V(z) P_{s}^{(o)}(z, w) V(w) P_{t-s-u}^{(o)}(z,-x) .
\end{aligned}
$$

By a direct integration, $\alpha=\frac{1}{2}$. Using $P_{t}^{(o)}(z,-x)=P_{t}^{(o)}(-z, x)$ and doing the $x$ integral:

$$
\beta=-t \int_{\mathrm{R}} d z P_{t}^{(o)}(z,-z) V(z)=-\frac{1}{2} t V(0)+o(t)
$$

if 0 is a point of Lebesgue continuity for $V$.

Thus the result is reduced to proving

$$
\gamma=o(t)
$$

Doing the $x$ integral as for $\beta$ :

$$
\gamma=\frac{1}{2} \int d z d w d s(t-s) P_{t-s}^{(o)}(z,-w) V(z) V(w) P_{s}^{(o)}(z, w)
$$

so it suffices to show that

$$
\delta \equiv \int d z d w d s P_{t-s}^{(o)}(z,-w)|V(z)||V(w)| P_{s}^{(o)}(z, w)=o(1)
$$

Write $\delta \leq \delta_{1}+\delta_{2}+\delta_{3}$ where $\delta_{1}$ is the integral over the region $|w-z|>\frac{1}{2} t^{1 / 4}, \delta_{2}$ the region where $|w+z|>\frac{1}{2} t^{1 / 4}$, and $\delta_{3}$ the region where $|w|<t^{1 / 4},|z|<t^{1 / 4}$. The $\delta_{1}, \delta_{2}$ integrals are bounded by $\left(\int_{[-1,1]}|V(z)|\right)^{2} e^{-c / t^{1 / 2}} \int_{0}^{t} d s(t-s)^{-1 / 2}\left(s^{-1 / 2}\right)=O\left(e^{-c / t^{1 / 2}}\right)=o(1)$ since

$$
\int_{0}^{t} d s(t-s)^{-1 / 2} s^{-1 / 2}=\int_{0}^{1} d s s^{-1 / 2}(1-s)^{-1 / 2}<\infty .
$$

To control $\delta_{3}$, bound $P_{u}^{(o)}$ by $C u^{-1 / 2}$ and find, by (3.7)

$$
\delta_{3}=C\left(\int_{|x|<t^{1 / 4}} d x|V(x)|\right)^{2} \int_{0}^{1} d s s^{-1 / 2}(1-s)^{-1 / 2}=o(1)
$$

since $V \in L^{1}(\mathrm{R})$ (w.l.o.g. $\left.\operatorname{supp}(V) \subset[-1,1]\right)$. 


\section{§4. Asymptotic Expansions}

Our goal in this section is to prove a number of related theorems on

$$
F(x, t):=\operatorname{Tr}\left(e^{-t H_{D ; x}}-e^{-t H}\right) .
$$

Theorem 4.1. Suppose that $V(x)$ is $C^{\infty}$ and bounded from below. Then $F(x, t)$ has an asymptotic expansion as $t \downarrow 0$ :

$$
F(x, t) \sim \sum_{j=0}^{\infty} s_{j}(x) t^{j}
$$

where $s_{j}(x)$ is dependent only on the numbers $V(x), \ldots, V^{(k)}(x)\left(V^{(k)}(x)\right):=\left(d^{k} V / d x^{k}(x)\right)$ with $k=2 j-2$.

Theorem 4.2. Suppose that $V(x)$ is bounded from below and locally bounded from above. Fix $x_{0}$ and $n$ and suppose that near $x_{0}$,

$$
V(x)=\sum_{j=0}^{2 n-2} b_{j}\left(x-x_{0}\right)^{j}+o\left(\left|x-x_{0}\right|^{2 n-2}\right) .
$$

Then there exists $\left\{s_{j}\left(x_{0}\right)\right\}_{j=0}^{n}$ such that

$$
F\left(x_{0}, t\right)=\sum_{j=0}^{n} s_{j}\left(x_{0}\right) t^{j}+o\left(t^{n}\right) .
$$

The $s_{j}\left(x_{0}\right)$ are the same functions of the b's as in Theorem 4.1.

Theorem 4.3. Suppose that $V(x)$ is $C^{\infty}$ and bounded from below and

$$
\left|V^{(k)}(x)\right| \leq C_{k} e^{A_{k} x^{2}}
$$

for some $C_{k}, A_{k}$. Then for $j \geq 1$

$$
\lim _{t \downarrow 0} \int_{0}^{\infty} d \lambda e^{-\lambda t} \lambda^{j-1}(\lambda t-j) \xi\left(\lambda, x_{0}\right)=(-1)^{j+1} s_{j}\left(x_{0}\right) j !
$$

and if $V \geq 0 ; j \geq 1$

$$
\lim _{t \downarrow 0} \int_{0}^{\infty} d \lambda \lambda^{j-1} e^{-\lambda t}\left(\xi\left(\lambda, x_{0}\right)-\frac{1}{2}\right)=(-1)^{j} s_{j}\left(x_{0}\right)(j-1) ! .
$$

Proof. Theorem 4.1 clearly follows from Theorem 4.2. The first assertion in Theorem 4.3 follows directly from

$$
F(x, t)=-t \int_{0}^{\infty} d \lambda e^{-\lambda t} \xi(\lambda, x)
$$


if we prove that $F(x, t)$ is $C^{\infty}$ in $t$ with derivatives having limits at $t=0$. The second equality then follows if we note that

$$
\left.F(x, t)\right|_{V \equiv 0}=-\frac{1}{2} t \int_{0}^{\infty} d \lambda e^{-\lambda t}
$$

SO

$$
\sum_{j=1}^{\infty} s_{j}\left(x_{0}\right) t^{j-1} \sim-\int_{0}^{\infty} d \lambda e^{-\lambda t}\left(\xi\left(\lambda, x_{0}\right)-\frac{1}{2}\right) .
$$

Thus the proofs are reduced to showing Theorem 4.2 and that under the hypothesis (4.2), $F$ is $C^{\infty}$ in $t$ with continuous derivatives at $t=0$. W.l.o.g. take $x_{0}=0$.

We turn first to Theorem 4.2. As in the last section, let $W(x)=V(x) \chi_{[-1,1]}(x)$ and note that by Theorem 2.2

$$
\left.F(0, t)\right|_{V}-\left.F(0, t)\right|_{W}=O\left(e^{-C / t}\right)
$$

so we can suppose that $V$ is supported in $[-1,1]$ which we will henceforth do. By local boundedness, we can suppose $\|V\|_{\infty}<\infty$. Use an asymptotic expansion of

$$
F(0, t)=-\frac{1}{2} E_{\omega}\left(\exp \left(-t \int_{0}^{1} d s V(\sqrt{2 t} \omega(s))\right)\right)=\sum_{j=0}^{n} b_{j}(t)+R_{n}(t)
$$

where

$$
b_{j}(t)=\frac{(-1)^{j+1}}{2(j !)} E_{\omega}\left(t^{j}\left(\int_{0}^{1} d s V(\sqrt{2 t} \omega(s))\right)^{j}\right)
$$

and for $0 \leq t \leq 1$

$$
\left|R_{n}(t)\right| \leq \exp \left(\|V\|_{\infty}\right) t^{n+1}\|V\|_{\infty}^{n+1} /(n+1) !
$$

since Taylor's theorem with remainder implies

$$
\left|e^{-x}-\sum_{j=0}^{n}(-x)^{j} / j !\right| \leq C^{n+1} e^{C} /(n+1) !
$$

if $|x| \leq C$.

By hypothesis $V$ has an asymptotic expansion

$$
V(x)=\sum_{j=0}^{2 n-2} b_{j} x^{j}+o\left(x^{2 n-2}\right)
$$


Plug that into $b_{j}(t), j \geq 1$ and find

$$
b_{j}(t)=\frac{(-1)^{j+1}}{2(j !)} E_{\omega}\left(t^{j} \int_{0}^{1} d s \sum_{k=1}^{2 n-2} C_{k, j}\left(b_{1}, \ldots, b_{k}\right)(\sqrt{2 t})^{k}(\omega(s))^{k}\right)+o\left(t^{n+j-1}\right),
$$

where $C_{k, j}\left(b_{1}, \ldots, b_{k}\right)$ are certain polynomials in $b_{1}, \ldots, b_{k}$. Since $E\left(\omega(s)^{j}\right)=0$ if $j$ is odd (by $x \rightarrow-x$ symmetry), we have the required asymptotic series proving Theorem 4.2 .

As for Theorem 4.3, under hypothesis (4.2) define

$$
K(t):=F\left(x=0, t^{2}\right)
$$

Then there are formal formulae one can write down for $d^{\ell} K / d t^{\ell}$ by differentiating inside the $E(\cdots)$ expectation and integral. Because of (4.2), it is easy to see the integrand in $E(\cdots)$ converges absolutely, and then by integrating the derivative that the formal formula is valid. With this formula in hand, one sees that $d^{\ell} K / d t^{\ell}$ is continuous as $t \downarrow 0$ and $d^{\ell} K / d t^{\ell}=0$ if $\ell$ is odd. It follows by Taylor's theorem with remainder that

$$
K(t)=\sum_{j=0}^{n} a_{j} t^{2 j}+E_{n}(t)
$$

with

$$
\frac{d^{m} E_{n}(t)}{d t^{m}}=O\left(t^{2 n+1-m}\right), \quad m=0, \ldots, 2 l .
$$

But $F(x=0, t)=K(\sqrt{t})$. Using (4.4), F(t) has continuous derivatives at $t=0$, that is, we have proven what is used to conclude Theorem 4.3.

\section{$\S 5$. Analysis of the Coefficients}

In $\S 4$ we proved the existence of an asymptotic expansion of the form

$$
\operatorname{Tr}\left(e^{t H_{D ; x}}-e^{-t H}\right) \underset{t \downarrow 0}{\sim} \sum_{j=0}^{\infty} s_{j}(x) t^{j}, \quad x \in \mathrm{R}
$$

assuming

$$
V \in C^{\infty}(\mathrm{R}), \quad V \text { real-valued and bounded from below }
$$

so that the differential expression

$$
h=-\frac{d^{2}}{d x^{2}}+V(x), \quad x \in \mathrm{R}
$$

is non-oscillatory at $\pm \infty$ (and hence in the limit point case at $\pm \infty$ ). The main purpose of this section is to identify the coefficients $s_{j}(x), j \in \mathrm{N}$ in (5.1) with the KdV invariants (and hence with certain differential polynomials of $V$ ). 
In order to identify $s_{j}(x), j \in \mathrm{N}$ with the $\mathrm{KdV}$ invariants, we adopt the following strategy. By strengthening the assumptions (5.2), (5.3) momentarily to

$$
V \in C_{0}^{\infty}(\mathrm{R})
$$

we shall derive the asymptotic expansion

$$
\operatorname{Tr}\left[\left(H_{D ; x}-z\right)^{-1}-(H-z)^{-1}\right] \underset{z \downarrow-\infty}{\sim} \sum_{j=0}^{\infty} r_{j}(x) z^{-j-1}
$$

and relate $r_{j}(x), j \in \mathrm{N}$ with the $\mathrm{KdV}$ invariants by means of well-known Riccati-type equation arguments. The Laplace transform connecting (5.1) and (5.5) is then used to derive the relation

$$
s_{j}(x)=(-1)^{j+1}(j !)^{-1} r_{j}(x), \quad j \in \mathrm{N}_{0}=\mathrm{N} \cup\{0\}
$$

which then identifies $s_{j}(x)$ and the KdV invariants (up to inessential numerical factors). Since by Theorem 4.1, $s_{j}(x)$ only depends on the numbers $V(x), \ldots, V^{(k)}(x)$ with $k=$ $2 j-2$, the connection (5.6) between $s_{j}(x)$ and $r_{j}(x)$ is independent of the short-range nature of $V \in C_{0}^{\infty}(\mathrm{R})$ and extends to all $V \in C^{\infty}(\mathrm{R})$ bounded from below. In fact, it extends to $V$ bounded from below and locally bounded from above, satisfying the asymptotic expansion assumed in Theorem 4.2.

Theorem 5.1. Assume $V \in C_{0}^{\infty}(\mathrm{R})$. Then for each $N \in \mathrm{N}$,

$$
\begin{aligned}
\operatorname{Tr}\left[\left(H_{D ; x}-z\right)^{-1}-(H-z)^{-1}\right]=-\frac{d}{d z} & \ln [G(z, x, x)] \\
& \sim \sum_{z \downarrow-\infty}^{N} r_{j=0}(x) z^{-j-1}+O\left(z^{-N-1}\right), \quad x \in \mathrm{R}
\end{aligned}
$$

uniformly with respect to $x \in \mathrm{R}$, where $r_{j}(x), j \in \mathrm{N}$ represent the $K d V$ invariants. More precisely, one has

$$
\begin{gathered}
r_{0}(x)=\frac{1}{2}, \quad r_{1}(x)=\frac{1}{2} V(x), \\
r_{j}(x)=(-1)^{j+1} 2^{1-2 j} j \phi_{2 j-1}(x)+\sum_{\ell=1}^{j-1}(-1)^{j-\ell+1} 2^{1-2(j-\ell)} \phi_{2(j-\ell)-1}(x) r_{\ell}(x), \quad j \geq 2,
\end{gathered}
$$

where $\phi_{j}(x), j \in \mathrm{N}$ are given by the recursion relation

$$
\begin{gathered}
\phi_{1}(x)=V(x), \quad \phi_{2}(x)=-V^{\prime}(x), \\
\phi_{j+1}(x)=-\phi_{j}^{\prime}(x)-\sum_{\ell=1}^{j-1} \phi_{\ell}(x) \phi_{j-\ell}(x), \quad j \geq 2 .
\end{gathered}
$$


Proof. Since $V \in C_{0}^{\infty}(\mathrm{R})$, one can set up the Volterra integral equations

$$
\begin{gathered}
f_{ \pm}(z, x)=e^{ \pm i z^{1 / 2} x}+\int_{ \pm \infty}^{x} d x_{1} z^{-1 / 2} \sin \left[z^{1 / 2}\left(x-x_{1}\right)\right] V\left(x_{1}\right) f_{ \pm}\left(z, x_{1}\right) \\
\operatorname{Im}\left(z^{1 / 2}\right) \geq 0, \quad z \in \mathrm{C}, x \in \mathrm{R}
\end{gathered}
$$

such that

$$
H f_{ \pm}(z, x)=z f_{ \pm}(z, x), \quad z \in \mathrm{C}
$$

in the sense of distributions. Better suited for our purpose are actually $g_{ \pm}(z, x)$ defined by

$$
g_{ \pm}(z, x)=e^{\mp i z^{1 / 2} x} f_{ \pm}(z, x)
$$

satisfying

$$
g_{ \pm}(z, x)=1 \pm\left(2 i z^{1 / 2}\right)^{-1} \int_{ \pm \infty}^{x} d x_{1}\left[1-e^{\mp 2 i z^{1 / 2}\left(x-x_{1}\right)}\right] V\left(x_{1}\right) g_{ \pm}\left(z, x_{1}\right)
$$

Iterating (5.13) one infers by a standard procedure that

$$
\left|g_{ \pm}(z, x)\right| \leq C, \quad z \in \mathrm{C}, x \in \mathrm{R}
$$

and combined with integrations by parts, one obtains the asymptotic expansions

$$
g_{ \pm}^{(m)}(z, x) \underset{\operatorname{Im}\left(z^{1 / 2}\right) \geq 0}{\sim} \sum_{j=0}^{\infty} g_{ \pm, j}^{(m)}(x)\left(2 i z^{1 / 2}\right)^{-j}, \quad m \in \mathrm{N}_{0}, x \in \mathrm{R}
$$

uniformly with respect to $x \in \mathrm{R}$. In order to illustrate (5.15), it suffices to discuss as a special case the asymptotic expansion of $g_{ \pm}(z, x)$ up to order $O\left(z^{-2}\right)$. Using (5.13), one 
infers from repeated integrations by parts that

$$
\begin{aligned}
& g_{ \pm}(z, x)=1 \pm \frac{1}{2 i z^{1 / 2}} \int_{ \pm \infty}^{x} d x_{1} V\left(x_{1}\right) \mp \frac{e^{\mp 2 i z^{1 / 2} x}}{2 i z^{1 / 2}} \int_{ \pm \infty}^{x} d x_{1} e^{ \pm 2 i z^{1 / 2} x_{1}} V\left(x_{1}\right) \\
& +\frac{1}{\left(2 i z^{1 / 2}\right)^{2}} \int_{ \pm \infty}^{x} d x_{1}\left[1-e^{\mp 2 i z^{1 / 2}\left(x-x_{1}\right)}\right] V\left(x_{1}\right) \int_{ \pm \infty}^{x_{1}} d x_{2}\left[1-e^{\mp 2 i z^{1 / 2}\left(x_{1}-x_{2}\right)}\right] V\left(x_{2}\right) \\
& \pm \frac{1}{\left(2 i z^{1 / 2}\right)^{3}} \int_{ \pm \infty}^{x} d x_{1}\left[1-e^{\mp 2 i z^{1 / 2}\left(x-x_{1}\right)}\right] V\left(x_{1}\right) \int_{ \pm \infty}^{x_{1}} d x_{2}\left[1-e^{\mp 2 i z^{1 / 2}\left(x_{1}-x_{2}\right)}\right] V\left(x_{2}\right) \cdot \\
& \int_{ \pm \infty}^{x_{2}} d x_{3}\left[1-e^{\mp 2 i z^{1 / 2}\left(x_{2}-x_{3}\right)}\right] V\left(x_{3}\right) g_{ \pm}\left(z, x_{3}\right) \\
& \underset{\operatorname{Im}\left(z^{1 / 2}\right) \geq 0}{\sim} 1 \pm \frac{1}{2 i z^{1 / 2}} \int_{ \pm \infty}^{x} d x_{1} V\left(x_{1}\right)+\frac{1}{4 z} V(x)-\frac{1}{8 z}\left[\int_{ \pm \infty}^{x} d x_{1} V\left(x_{1}\right)\right]^{2} \\
& \mp \frac{1}{8 i z^{3 / 2}} V^{\prime}(x) \pm \frac{1}{8 i z^{3 / 2}} \int_{ \pm \infty}^{x} d x_{1} V\left(x_{1}\right)^{2} \pm \frac{1}{8 i z^{3 / 2}} V(x) \int_{ \pm \infty}^{x} d x_{1} V\left(x_{1}\right) \\
& \mp \frac{1}{64 i z^{3 / 2}}\left[\int_{ \pm \infty}^{x} d x_{1} V\left(x_{1}\right)\right]^{3}+O\left(z^{-2}\right), \quad x \in \mathrm{R},
\end{aligned}
$$

where we used (5.14) to arrive at the $O\left(z^{-2}\right)$-term uniformly with respect to $x \in \mathrm{R}$. By induction one extends this expansion to $O\left(z^{-N}\right)$ for each $N \in \mathrm{N}$ uniformly in $x \in$ R. Analogously, one arrives at the corresponding expansions for $g^{(m)}(z, x), m \in \mathrm{N}$. In particular, introducing

$$
\phi_{ \pm}(z, x)=\frac{f_{ \pm}^{\prime}(z, x)}{f_{ \pm}(z, x)}= \pm i z^{1 / 2}+\frac{g_{ \pm}^{\prime}(z, x)}{g_{ \pm}(z, x)}
$$

( ${ }^{\prime}$ denotes $\left.d / d x\right)$ one obtains

$$
\phi_{ \pm}^{(m)}(z, x) \underset{\substack{z \rightarrow \infty \\ \operatorname{Im}\left(z^{1 / 2}\right) \geq 0}}{\sim} \pm i z^{1 / 2}+\sum_{j=1}^{\infty} \phi_{ \pm}^{(m)}(x)\left(2 i z^{1 / 2}\right)^{-j}, \quad x \in \mathrm{R}
$$

for certain coefficients $\phi_{ \pm}(x)$ (uniformly in $x \in \mathrm{R}$ since $V \in C_{0}^{\infty}(\mathrm{R})$ ). Combining (5.11), (5.12), and (5.17) yields the Riccati-type equation

$$
\phi_{ \pm}^{\prime}(z, x)+\phi_{ \pm}(z, x)^{2}=V(x)-z
$$


A comparison of (5.18) and (5.19) then yields

$$
\begin{gathered}
\phi_{ \pm, 1}(x)= \pm V(x), \quad \phi_{ \pm, 2}(x)=-V^{\prime}(x), \\
\phi_{ \pm, j+1}(x)=\mp \phi_{ \pm, j}^{\prime}(x) \mp \sum_{\ell=1}^{j-1} \phi_{ \pm, \ell}(x) \phi_{ \pm, j-\ell}(x), \quad j \geq 2 .
\end{gathered}
$$

This identifies $\phi_{+, j}$ and $\phi_{j}$

$$
\phi_{+, j}(x)=\phi_{j}(x), \quad j \in \mathrm{N}
$$

as introduced in (5.9) and also yields

$$
\phi_{-, j}(x)=(-1)^{j} \phi_{+, j}(x), \quad j \in \mathrm{N} .
$$

In order to connect (5.7) and (5.18) we recall a few facts. First of all, the Green's function $G\left(z, x, x^{\prime}\right)$ of $H$ satisfies

$$
G(z, x, x)=\left[\phi_{-}(z, x)-\phi_{+}(z, x)\right]^{-1}, \quad z \in \mathrm{C} \backslash \operatorname{spec}(H), x \in \mathrm{R}
$$

since, due to definition (5.17), $\phi_{ \pm}(z, x)$ equal the Weyl $m$-functions associated with $H_{D, \pm ; x}$ in $L^{2}((x, \pm \infty))$, the restrictions of $H$ to $(x, \pm \infty)$ with a Dirichlet boundary condition at $x \in \mathrm{R}$. In particular,

$$
H_{D ; x}=H_{D,-; x} \oplus H_{D,+; x} .
$$

Thus we obtain

$$
\begin{aligned}
\operatorname{Tr}\left[\left(H_{D ; x}-z\right)^{-1}\right. & \left.-(H-z)^{-1}\right]=-\frac{d}{d z} \ln [G(z, x, x)]=\frac{d}{d z} \ln \left[\phi_{-}(z, x)-\phi_{+}(z, x)\right] \\
& \sim \underset{z \downarrow-\infty}{\sim} \frac{d}{d z} \ln \left[z^{1 / 2} \sum_{j=0}^{\infty} 2 \phi_{2 j-1}(x)\left(2 i z^{1 / 2}\right)^{-2 j}\right] \\
& \sim \underset{z \downarrow-\infty}{\sim}(1 / 2 z)+\frac{d}{d z} \ln \left[1+\sum_{j=1}^{\infty}(-1)^{j} 2^{1-2 j} \phi_{2 j-1}(x) z^{-j}\right] \\
& \sim \underset{z \downarrow-\infty}{\sim} \sum_{j=0}^{\infty} r_{j}(x) z^{-j-1}
\end{aligned}
$$

where $r_{j}(x), j \in \mathrm{N}_{0}$ are given by (5.8). Here we made use of (5.23), (5.18), (5.22), and the fact that if $F$ has the asymptotic expansion

$$
F(z) \underset{|z| \rightarrow \infty}{\sim} \sum_{j=1}^{\infty} c_{j} z^{-j}
$$


then

$$
\ln [1+F(z)] \underset{|z| \rightarrow \infty}{\sim} \sum_{j=1}^{\infty} d_{j} z^{-j}
$$

where

$$
\begin{aligned}
& d_{1}=c_{1}, \\
& d_{j}=c_{j}-\sum_{\ell=1}^{j-1}(\ell / j) c_{j-\ell} d_{\ell}, \quad j \geq 2 .
\end{aligned}
$$

Remark 5.2. (i) Using

$$
G(z, x, x)=\frac{f_{+}(z, x) f_{-}(z, x)}{W\left(f_{+}(z), f_{-}(z, x)\right)}, \quad z \in \mathrm{C} \backslash \operatorname{spec}(H), x \in \mathrm{R}
$$

one derives the relation (see, e.g., [27])

$$
\frac{d}{d x} \ln [G(z, x, x)]=\phi_{-}(z, x)+\phi_{+}(z, x)
$$

which yields the simpler expression

$$
\begin{aligned}
\frac{d}{d x} \operatorname{Tr}\left[\left(H_{D ; x}-z\right)^{-1}-(H-z)^{-1}\right] & =\frac{d}{d z} \frac{d}{d x} \ln \left[\phi_{-}(z, x)-\phi_{+}(z, x)\right] \\
& =-\frac{d}{d z}\left[\phi_{+}(z, x)+\phi_{-}(z, x)\right] \underset{z \downarrow-\infty}{\sim} \sum_{j=1}^{\infty}(-1)^{j} 2^{1-2 j} \phi_{2 j}(x) z^{-j-1} .
\end{aligned}
$$

Integrating (5.32) term by term (putting integration constants indentically zero since $r_{j}(x)$ are homogeneous differential polynomials of degree $\operatorname{deg}\left(r_{j}\right)=2 j, j \in \mathrm{N}_{0}$ defining $\operatorname{deg}\left(V^{(m)}\right)=m+2, m \in \mathrm{N}_{0}$ ) yields (5.7) except for the leading term $1 / 2 z$ which can be inferred from the free case $V^{(o)}(x) \equiv 0$.

(ii) Relations (5.22) and (5.31) prove that $\phi_{ \pm, 2 m}(x)$ are total derivatives, that is,

$$
\phi_{ \pm, 2 m}(x)=\frac{d}{d x} \eta_{m}(x), \quad m \in \mathrm{N}
$$

for some differential polynomials $\eta_{m}$ of $V$ with $\eta_{m} \in C_{0}^{\infty}(\mathrm{R})\left(\operatorname{resp} . C^{\infty}(\mathrm{R})\right)$ if $V \in C_{0}^{\infty}(\mathrm{R})$ (resp. $C^{\infty}(\mathrm{R})$ ). Moreover, the following asymptotic expansion holds (see, e.g., [12], [13]).

$$
G(z, x, x) \underset{z \downarrow-\infty}{\sim}-\sum_{j=0}^{\infty} \omega_{2 j}(x)\left(2 i z^{1 / 2}\right)^{-2 j-1}, \quad x \in \mathrm{R}
$$

uniformly with respect to $x \in \mathrm{R}$, where

$$
\begin{aligned}
\omega_{0}(x) & =1 \\
\omega_{2 j}(x) & =-2 \phi_{2 j-1}(x)-2 \sum_{\ell=1}^{j-1} \phi_{2 \ell-1}(x) \omega_{2(j-\ell)}(x), \quad j \in \mathrm{N}
\end{aligned}
$$


One can prove that $([10],[13])$

$$
\omega_{2 j+2}(x)=-2(2 j+1) \phi_{2 j+1}(x)+\frac{d}{d x} \nu_{j}(x), \quad j \in \mathrm{N}_{0},
$$

where $\nu_{j}$ are differential polynomials in $V$ with $\nu_{j} \in C_{0}^{\infty}(\mathrm{R})\left(\operatorname{resp} . C^{\infty}(\mathrm{R})\right)$ if $V \in C_{0}^{\infty}(\mathrm{R})$ (resp. $\left.V \in C^{\infty}(\mathrm{R})\right)$.

(iii) Clearly (5.7), (5.32), and (5.33) extend to uniformly asymptotic expansions as $|z| \rightarrow \infty$ outside any cone with apex $E_{o}=\inf \operatorname{spec}(H)$ and arbritrarily small opening angle $\epsilon>0$ along the positive real axis. Explicitly, one infers from (5.8), (5.9), and (5.35) that

$$
\begin{gathered}
r_{0}(x)=\frac{1}{2}, \quad r_{1}(x)=\frac{1}{2} V(x), \quad r_{2}(x)=\frac{1}{2} V(x)^{2}-\frac{1}{4} V^{\prime \prime}(x), \quad \text { etc. } \\
\phi_{1}(x)=V(x), \quad \phi_{2}(x)=-V^{\prime}(x), \quad \phi_{3}(x)=V^{\prime \prime}(x)-V(x)^{2} \\
\phi_{4}(x)=4 V(x) V^{\prime}(x)-V^{\prime \prime \prime}(x), \quad \text { etc. }
\end{gathered}
$$

and

$$
\omega_{0}(x)=1, \quad \omega_{2}(x)=-2 V(x), \quad \omega_{4}(x)=6 V(x)^{2}-2 V^{\prime \prime}(x), \quad \text { etc. }
$$

Next we relate $(5.7)$ and $(5.1)$.

Theorem 5.3. Suppose $V \in C^{\infty}(\mathrm{R}), V$ real-valued and bounded from below. Then for each $N \in \mathrm{N}$,

$$
\operatorname{Tr}\left(e^{-t H_{D ; x}}-e^{-t H}\right) \underset{t \downarrow 0}{\sim} \sum_{j=0}^{N} s_{j}(x) t^{j}+O\left(t^{N+1}\right), \quad x \in \mathrm{R},
$$

where $s_{j}(x)$ are the $K d V$ invariants

$$
s_{j}(x)=(-1)^{j+1}(j !)^{-1} r_{j}(x), \quad j \in \mathrm{N}_{0}
$$

with $r_{j}(x)$ given by $(5.8)$.

Proof. Since the existence of the asymptotic expansion (5.40) has been proven in $\S 4$ we only need to identify the coefficients $s_{j}(x)$ as in (5.41). Without loss of generality we may assume in addition that $V \in C_{0}^{\infty}(\mathrm{R})$. Let $E_{o}=\inf \operatorname{spec}(H)$, then one obtains from (1.4) and Fubini's theorem that

$$
\begin{aligned}
\operatorname{Tr}\left[\left(H_{D ; x}-z\right)^{-1}-(H-z)^{-1}\right] & =-\int_{E_{o}}^{\infty} \frac{d \lambda \xi(\lambda, x)}{(\lambda-z)^{2}} \\
& =\int_{E_{o}}^{\infty} d \lambda \xi(\lambda, x) \int_{0}^{\infty} d t(-t) e^{(z-\lambda) t} \\
& =\int_{0}^{\infty} d t e^{z t} \int_{E_{o}}^{\infty} d \lambda(-t) e^{-\lambda t} \xi(\lambda, x) \\
& =\int_{0}^{\infty} d t e^{z t} \operatorname{Tr}\left(e^{-t H_{D ; x}}-e^{-t H}\right), \quad z<E_{o} .
\end{aligned}
$$


Define

$$
F(x, t)=\operatorname{Tr}\left(e^{-t H_{D ; x}}-e^{-t H}\right)=-t \int_{E_{o}}^{\infty} d \lambda e^{-t \lambda} \xi(\lambda, x), \quad t>0, x \in \mathrm{R} .
$$

Then

$$
F(x, \cdot) \in C^{\infty}([0, \infty)), \quad \text { for each } x \in \mathrm{R}
$$

is proven at the end of $\S 4$ and Theorem 4.1 yields for each $N \in \mathrm{N}$,

$$
F(x, t) \underset{t \downarrow 0}{\sim} \sum_{j=0}^{N} s_{j}(x) t^{j}+O\left(t^{N+1}\right)
$$

In particular,

$$
\left|F(x, t)-\sum_{j=0}^{N} s_{j}(x) t^{j}\right| \leq C_{N}(x) t^{N+1}, \quad 0 \leq t \leq 1
$$

by estimating the remainder in the Taylor expansion for $F(x, \cdot)$. Thus

$$
z \operatorname{Tr}\left[\left(H_{D ; x}-z\right)^{-1}-(H-z)^{-1}\right]=z \int_{0}^{1} d t e^{z t} F(x, t)+z \int_{1}^{\infty} d t e^{z t} F(x, t):=G_{1}(x, z)+G_{2}(x, z) .
$$

Clearly,

$$
\left|G_{2}(x, z)\right|=\left|z \int_{1}^{\infty} d t e^{z t} F(x, t)\right| \leq(-z) \int_{1}^{\infty} d t e^{z t}|F(x, t)| \leq C_{0} e^{z}, \quad z<\min \left(0, E_{o}\right)
$$

since $|F(x, t)| \leq e^{-t E_{o}}$ (because of $0 \leq \xi(\lambda, x) \leq 1$ ). Moreover,

$$
\begin{aligned}
& G_{1}(x, z)=z \int_{0}^{1} d t e^{z t}\left[F(x, t)-\sum_{j=0}^{N} s_{j}(x) t^{j}+\sum_{j=0}^{N} s_{j}(x) t^{j}\right] \\
& \sim \sum_{z \downarrow-\infty}^{N} s_{j=0}(x)\left[z \int_{0}^{\infty} d t e^{z t} t^{j}+O\left(e^{\epsilon z}\right)\right]+z \int_{0}^{1} d t e^{z t}\left[F(x, t)-\sum_{j=0}^{N} s_{j}(x) t^{j}\right] \\
& \underset{z \downarrow-\infty}{\sim} \sum_{j=0}^{N} s_{j}(x)(-1)^{j+1}(j !)\left[z^{-j}+O\left(e^{\epsilon z}\right)\right]+z \int_{0}^{1} d t e^{z t}\left[F(x, t)-\sum_{j=0}^{N} s_{j} t^{j}\right], \quad z<\min \left(0, E_{o}\right)
\end{aligned}
$$


for some $0<\epsilon<1$. Thus

$$
z \operatorname{Tr}\left[\left(H_{D ; x}-z\right)^{-1}-(H-z)^{-1}\right] \underset{z \downarrow-\infty}{\sim} \sum_{j=0}^{N} s_{j}(x)(-1)^{j+1}(j !) z^{-j}+O\left(z^{-N-1}\right)
$$

using the estimate (5.46). A comparison of (5.7) and (5.50) then yields (5.41).

Relations (5.37) and (5.41) then yield explicitly

$$
s_{0}(x)=-\frac{1}{2}, \quad s_{1}(x)=\frac{1}{2} V(x), \quad s_{2}(x)=\frac{1}{8} V^{\prime \prime}(s)-\frac{1}{4} V(x)^{2}, \quad \text { etc. }
$$

Finally we epxress the $\mathrm{KdV}$ invariants $s_{j}(x)$ in terms of $\xi(\lambda, x)$ according to Theorem 4.3 .

Theorem 5.4. Suppose $V \in C^{\infty}(\mathrm{R}), V$ real-valued and bounded from below. Assume that (4.2) holds and denote $E_{o}=\inf \operatorname{spec}(H)$. Then

$$
\begin{aligned}
& s_{0}(x)=-\frac{1}{2}, \\
& s_{j}(x)=\frac{(-1)^{j+1}}{j !}\left\{\frac{E_{o}^{j}}{2}+j \lim _{t \downarrow 0} \int_{E_{o}}^{\infty} d \lambda e^{-t \lambda} \lambda^{j-1}\left[\frac{1}{2}-\xi(\lambda, x)\right] \quad j \in \mathrm{N}, x \in \mathrm{R} .\right.
\end{aligned}
$$

Explicitly, one has

$$
\begin{aligned}
s_{1}(x) & =\frac{1}{2} V(x) \\
& =\frac{E_{o}}{2}+\lim _{t \downarrow 0} \int_{E_{o}}^{\infty} d \lambda e^{-t \lambda}\left[\frac{1}{2}-\xi(\lambda, x)\right], \\
s_{2}(x) & =\frac{1}{8} V^{\prime \prime}(x)-\frac{1}{4} V(x)^{2} \\
& =-\frac{E_{o}^{2}}{4}-\lim _{t \downarrow 0} \int_{E_{o}}^{\infty} d \lambda e^{-t \lambda} \lambda\left[\frac{1}{2}-\xi(\lambda, x)\right], \quad \text { etc. }
\end{aligned}
$$

We will illustrate these results in the special case where $V(x)$ is periodic.

Example 5.5. Assume $V \in C^{\infty}(\mathrm{R}), V$ real-valued, for some $a>0, V(x+a)=V(x)$ for all $x \in \mathrm{R}$. In this case the spectrum of $H$ is given by

$$
\operatorname{spec}(H)=\bigcup_{n=1}^{\infty}\left[E_{2(n-1)}, E_{2 n-1}\right]
$$


Then for each $x \in \mathrm{R}, \xi(\lambda, x)$ is real-valued for $\lambda \in\left(E_{2 n-1}, E_{2 n}\right)$ and purely imaginary for $\lambda \in\left(E_{2(n-1)}, E_{2 n-1}\right)$ (see, e.g., [4], [28]). More precisely,

$$
\xi(\lambda, x)= \begin{cases}0, & \lambda<E_{0}, \quad \mu_{n}(x)<\lambda<E_{2 n}, \quad n \in \mathrm{N} \\ 1, & E_{2 n-1}<\lambda<\mu_{n}(x), \quad n \in \mathrm{N} \\ \frac{1}{2}, & E_{2(n-1)}<\lambda<E_{2 n-1}, \quad n \in \mathrm{N}\end{cases}
$$

(and analogously for limiting cases where $\mu_{n}(x) \in\left\{E_{2 n-1}, E_{2 n}\right\}, n \in \mathrm{N}$ ). Here $\mu_{n}(x)$ denote the Dirichlet eigenvalues (or limits thereof) of $H_{D ; x}$, that is,

$$
\operatorname{spec}\left(H_{D ; x}\right)=\left\{\mu_{n}(x)\right\}_{n \in \mathbb{N}} \cup \bigcup_{n=1}^{\infty}\left[E_{2(n-1)}, E_{2 n-1}\right], \quad E_{2 n-1} \leq \mu_{n}(x) \leq E_{2 n}, n \in \mathrm{N} .
$$

Inserting (5.56) into (5.52) and noticing that

$$
\left|E_{2 n}-E_{2 n-1}\right| \underset{n \rightarrow \infty}{=} 0\left(n^{-k}\right) \quad \text { for all } k \in \mathrm{N}
$$

since $V \in C^{\infty}$ (see [36], [40] and the references therein), one can interchange the limit $t \downarrow 0$ and the integral in (5.52) to obtain

$$
2(-1)^{j+1} j ! s_{j}(x)=2 r_{j}(x)=E_{0}^{j}+\sum_{n=1}^{\infty}\left[E_{2 n-1}^{j}+E_{2 n}^{j}-2 \mu_{n}(x)^{j}\right], \quad j \in \mathrm{N}, x \in \mathrm{R} .
$$

The periodic trace formula (5.59) for $j=1$ has been noticed by Hochstadt [25] and Dubrovin [7]. The general case $j \in \mathrm{N}$ appeared in McKean and van Moerbeke [35] and Flaschka [8]. For more recent accounts, see, for example, [2], [29], [32], [33], [40].

Remark 5.6. The heat kernel approach in $\S 2-4$ naturally leads to the heat kernel regularization for $s_{j}(x)$ in Theorem 5.4. Alternatively, we could have exploited a resolvent regularization for $r_{j}(x)$ as follows. Applying (1.4) to $f(\lambda)=(\lambda-z)^{-1}$ and expanding in $z^{-1}$ near $z^{-1}=0$ yields

$$
\begin{aligned}
\operatorname{Tr}\left[\left(H_{D ; x}-z\right)^{-1}-(H-z)^{-1}\right] & =-\int_{E_{o}}^{\infty} \frac{d \lambda \xi(\lambda, x)}{(\lambda-z)^{2}} \\
& =\frac{1}{2 z}-\frac{E_{o}}{2 z\left(E_{o}-z\right)}+\int_{E_{o}}^{\infty} \frac{d \lambda\left[\frac{1}{2}-\xi(\lambda, x)\right]}{(\lambda-z)^{2}} \\
& \sim \underset{z \rightarrow i \infty}{\sim} \frac{1}{2 z}+\sum_{j=1}^{\infty} r_{j}(x) z^{-j-1},
\end{aligned}
$$

where

$$
r_{j}(x)=\frac{E_{o}^{j}}{2}-\lim _{z \rightarrow i \infty} \int_{E_{o}}^{\infty} d \lambda \frac{z^{j+1}}{(\lambda-z)^{j+1}} j(-\lambda)^{j-1}\left[\frac{1}{2}-\xi(\lambda, x)\right], \quad j \in \mathrm{N}, x \in \mathrm{R}
$$


under the assumptions on $V$ as in Theorem 5.4. In particular,

$$
\begin{aligned}
r_{1}(x) & =\frac{1}{2} V(x) \\
& =\frac{E_{o}}{2}+\lim _{z \rightarrow i \infty} \int_{E_{o}}^{\infty} d \lambda \frac{z^{2}}{(\lambda-z)^{2}}\left[\frac{1}{2}-\xi(\lambda, x)\right] .
\end{aligned}
$$

We shall return to a detailed discussion of resolvent regularization (proving the existence of an asymptotic expansion of the type (5.7) under the hypothesis on $V$ as in Theorem 5.4) in $\S 6$ in connection with other self-adjoint boundary conditions different from the Dirichlet boundary condition at $x \in \mathrm{R}$.

\section{$\S 6$. Other Boundary Conditions}

In this section we shall study higher order trace formulas associated to boundary conditions other than the Dirichlet conditions studied so far. In general, we want to consider operators which decompose into a direct sum under the decomposition $L^{2}(-\infty, y) \oplus L^{2}(y, \infty)$ and which differ from $H$ by a rank one perturbation. It can be shown the later condition forces the boundary conditions to match, that is, in (6.1) below the boundary conditions

$$
g^{\prime}(y \pm 0)+\beta_{ \pm} g(y \pm 0)=0
$$

have $\beta_{+}=\beta_{-}$. Thus, we define

$$
\begin{gathered}
H_{\beta ; y} f=h f, \quad h=-\frac{d^{2}}{d x^{2}}+V(x), \quad x \in \mathrm{R} \\
\mathcal{D}\left(H_{\beta ; y}\right)=\left\{g \in \mathrm{L}^{2}(\mathrm{R}) \mid g, g^{\prime} \in A C_{\mathrm{loc}}(\mathrm{R} \backslash\{y\}), h g \in L^{2}(\mathrm{R}),\right. \\
\left.\lim _{\epsilon \downarrow 0}\left[g^{\prime}(y \pm \epsilon)+\beta g(y \pm \epsilon)\right]=0\right\}, \quad \beta \in \mathrm{R}, y \in \mathrm{R},
\end{gathered}
$$

where we assume again that $V$ satisfies

$$
V \in C^{\infty}(\mathrm{R}), V \text { real-valued and bounded from below. }
$$

Thus $H_{\beta=0 ; y}=H_{N ; y}$ represents a Neumann boundary condition at $y \in \mathrm{R}$ and formally, $H_{\beta=\infty ; y}=H_{D ; y}$. In analogy to

$$
\begin{aligned}
\left(H_{D ; y}-z\right)^{-1}=(H-z)^{-1}-G(z, y, y)^{-1}(\overline{G(z, y, \cdot)}, \cdot) G(z, \cdot, y), \\
z \in C \backslash\left\{\operatorname{spec}\left(H_{D ; y}\right) \cup \operatorname{spec}(H)\right\},
\end{aligned}
$$

one now obtains

$$
\begin{aligned}
& \left(H_{\beta ; y}-z\right)^{-1}=(H-z)^{-1}-\left[\left(\beta+\partial_{1}\right)\left(\beta+\partial_{2}\right) G(z, y, y)\right]^{-1} . \\
& \cdot\left(\overline{\left(\beta+\partial_{1}\right) G(z, y, \cdot)}, \cdot\right)\left(\beta+\partial_{2}\right) G(z, ., y), \quad z \in \mathrm{C} \backslash\left\{\operatorname{spec}\left(H_{\beta ; y}\right) \cup \operatorname{spec}(H)\right\}, \beta \in \mathrm{R} .
\end{aligned}
$$


Here

$$
\begin{gathered}
\partial_{1} G\left(z, y, x^{\prime}\right):=\left.\partial_{x} G\left(z, x, x^{\prime}\right)\right|_{x=y}, \quad \partial_{2} G(z, x, y):=\left.\partial_{x^{\prime}} G\left(z, y, x^{\prime}\right)\right|_{x^{\prime}=y} \\
\partial_{1} \partial_{2} G(z, x, y):=\left.\partial_{x} \partial_{x^{\prime}} G\left(z, x, x^{\prime}\right)\right|_{x=x^{\prime}=y}, \quad \text { etc. }
\end{gathered}
$$

and we note that

$$
\partial_{1} G(z, y, x)=\partial_{2} G(z, x, y), \quad x \neq y
$$

renders the rank-one piece self-adjoint in (6.4) for $z<\inf \operatorname{spec}\left(H_{\beta ; y}\right)$. Hence the Herglotz function $G(z, y, y)$ is now replaced by $\left[\left(\beta+\partial_{1}\right)\left(\beta+\partial_{2}\right) G(z, y, y)\right]$. The latter is Herglotz too as can be inferred from the first resolvent equation

$$
\partial_{x}^{r} \partial_{x^{\prime}}^{s} \operatorname{Im}\left[G\left(z, x, x^{\prime}\right)\right]=\int_{\mathrm{R}} d x^{\prime \prime}\left[\overline{\partial_{x}^{r} G\left(z, x, x^{\prime \prime}\right)}\right]\left[\partial_{x^{\prime}}^{s} G\left(z, x^{\prime \prime}, x^{\prime}\right)\right], \quad r, s \in\{0,1\}
$$

implying (together with (6.6))

$$
\begin{aligned}
& \operatorname{Im}\left[\left(\beta+\partial_{1}\right)\left(\beta+\partial_{2}\right) G(z, y, y,)\right] \\
& =\operatorname{Im}(z)\left\{\beta^{2} \int_{\mathrm{R}} d x^{\prime \prime}\left|G\left(z, x^{\prime \prime}, y\right)\right|^{2}+\beta \int_{\mathrm{R}} d x^{\prime \prime}\left[\overline{\partial_{1} G\left(z, y, x^{\prime \prime}\right)}\right] G\left(z, x^{\prime \prime}, y\right)\right. \\
& \left.\quad+\beta \int_{\mathrm{R}} d x^{\prime \prime} \overline{G\left(z, y, x^{\prime \prime}\right)}\left[\partial_{1} G\left(z, y, x^{\prime \prime}\right)\right]+\int_{\mathrm{R}} d x^{\prime \prime}\left|\partial_{1} G\left(z, y, x^{\prime \prime}\right)\right|^{2}\right\} \\
& \geq \operatorname{Im}(z)\left[\left\|\partial_{1} G(z, \cdot, y)\right\|_{2}-|\beta|\|G(z, \cdot, y)\|_{2}\right]^{2}>0 \quad \text { for } \operatorname{Im}(z)>0
\end{aligned}
$$

by Cauchy's inequality. Equation (5.25) then turns into

$$
\operatorname{Tr}\left[\left(H_{\beta ; x}-z\right)^{-1}-(H-z)^{-1}\right]=-\frac{d}{d z} \ln \left[\left(\beta+\partial_{1}\right)\left(\beta+\partial_{2}\right) G(z, x, x,)\right] \quad \beta \in \mathrm{R}, x \in \mathrm{R} .
$$

In order to introduce $\xi_{\beta}(\lambda, x)$, Krein's spectral shift function associated with the pair $\left(H_{\beta ; x}, H\right)$ (in analogy to $\xi(\lambda, x) \equiv \xi_{\infty}(\lambda, x)$ associated with $\left.\left(H_{D ; x} \equiv H_{\infty ; x}, H\right)\right)$, we next investigate $\left[\left(\beta+\partial_{1}\right)\left(\beta+\partial_{2}\right) G(z, x, x)\right]$ a bit further. First of all we notice that

$$
H_{\beta ; x} \leq H, \quad \beta \in \mathrm{R}, x \in \mathrm{R}
$$

as opposed to

$$
H_{D ; x}=H_{\infty ; x} \geq H, \quad x \in \mathrm{R} .
$$

One way of understanding (6.10) is in terms of quadratic forms. Let $Q\left(H_{\beta=0}\right)=N_{y}$ be the form domain of the Neumann boundary condition object. Then $\varphi$ 's in $N_{y}$ are continuous on $\mathrm{R} \backslash\langle y\rangle$ and have continuous boundary values $\varphi(y \pm 0) . Q\left(H_{\beta}\right)=N_{y}$ with

$$
\left(\varphi, H_{\beta} \varphi\right)=\left(\varphi, H_{\beta=0} \varphi\right)-\beta\left[|\varphi(y+)|^{2}-|\varphi(y-)|^{2}\right] .
$$


Let $N_{y}^{0}=\{\varphi \in N \mid \varphi(y+)=\varphi(y-)\}$. Thus $H$ is just the form $H_{\beta}$ restricted to $N_{y}^{0}$, so $H_{\beta, y} \leq H$.

Moroever, one easily verifies the identity

$$
\begin{aligned}
& {\left[\left(\beta+\partial_{1}\right)\left(\beta+\partial_{2}\right) G(z, x, x)\right]=\beta^{2} G(z, x, x)} \\
& +\beta\left[\frac{d}{d x} G(z, x, x)\right]+H(z, x, x), \quad z \in \mathrm{C} \backslash \mathrm{R}, \beta \in \mathrm{R}, x \in \mathrm{R},
\end{aligned}
$$

where

$$
H(z, x, x)=\frac{f_{+}^{\prime}(z, x) f_{-}^{\prime}(z, x)}{W\left(f_{+}(z), f_{-}(z)\right)}
$$

and

$$
\frac{d}{d x} H(z, x, x)=[V(x)-z] \frac{d}{d x} G(z, x, x)
$$

From

$$
G(z, x, x) \underset{z \downarrow-\infty}{=} \frac{1}{2|z|^{1 / 2}}+o\left(|z|^{-1 / 2}\right)
$$

in accordance with

$$
G(z, x, x)>0 \quad \text { for } z<\inf \operatorname{spec}(H),
$$

and from (6.14) one infers

$$
\begin{aligned}
H(z, x, x) & =H\left(z, x_{o}, x_{o}\right)+\int_{x_{o}}^{x} d x^{\prime}\left[V\left(x^{\prime}\right)-z\right]\left[\frac{d}{d x^{\prime}} G\left(z, x^{\prime}, x^{\prime}\right)\right] \\
& =H\left(z, x_{o}, x_{o}\right)+o\left(|z|^{1 / 2}\right) \\
z \downarrow-\infty & H(-\infty)
\end{aligned}
$$

upon integration by parts. In particular, the leading asymptotic behavior of $H(z, x, x)$ as $z \downarrow-\infty$ is independent of $x$ and can be obtained from the free case $V^{(o)}(x) \equiv 0$. Since for $V^{(o)}(x)=0$,

$$
G^{(o)}(z, x, x)=\frac{i}{2 z^{1 / 2}}, \quad H^{(o)}(z, x, x)=\frac{i z^{1 / 2}}{2}
$$

one infers

$$
H(z, x, x) \underset{z \downarrow-\infty}{=}-\frac{|z|^{1 / 2}}{2}+o\left(|z|^{1 / 2}\right)
$$

and hence

$$
\left[\left(\beta+\partial_{1}\right)\left(\beta+\partial_{2}\right) G(z, x, x)\right]<0 \quad \text { for }-z>0 \text { large enough. }
$$

Thus the exponential Herglotz representation [1] for $\left[\left(\beta+\partial_{1}\right)\left(\beta+\partial_{2}\right) G(z, x, x)\right]$ yields

$$
\left[\left(\beta+\partial_{1}\right)\left(\beta+\partial_{2}\right) G(z, x, x)\right]=\exp \left\{c+\int_{\mathrm{R}}\left[\frac{1}{\lambda-z}-\frac{\lambda}{1+\lambda^{2}}\right]\left[\xi_{\beta}(\lambda, x)+1\right]\right\} d \lambda
$$


for some $c \in \mathrm{R}$, where for each $x \in \mathrm{R}$ and a.e. $\lambda \in \mathrm{R}$

$$
\xi_{\beta}(\lambda, x)=\frac{1}{\pi} \lim _{\epsilon \downarrow 0} \operatorname{Im}\left\{\ln \left[\left(\beta+\partial_{1}\right)\left(\beta+\partial_{2}\right) G(\lambda+i \epsilon, x, x)\right]\right\}-1
$$

and

$$
-1 \leq \xi_{\beta}(\lambda, x) \leq 0, \quad \text { a.e. } \lambda \in \mathrm{R}, \quad \xi_{\beta}(\lambda, x)=0 \quad \lambda<\inf \operatorname{spec}\left(H_{\beta ; x}\right)
$$

in agreement with (6.10) and (6.20). Hence

$$
\operatorname{Tr}\left[f\left(H_{\beta ; x}\right)-f(H)\right]=\int_{\mathrm{R}} d \lambda f^{\prime}(\lambda) \xi_{\beta}(\lambda, x)
$$

for any $f \in C^{2}(\mathrm{R})$ with $\left(1+\lambda^{2}\right) f^{(j)} \in L^{2}((0, \infty)), j=1,2$ and for $f(\lambda)=(\lambda-z)^{-1}$, $z \in \mathrm{C} \backslash\left[\inf \operatorname{spec}\left(H_{\beta ; x}\right), \infty\right)$.

The following example in the free case $V^{(o)}(x) \equiv 0$ illustrates these facts.

Example 6.1. $V^{(o)}(x) \equiv 0$. Then $G^{(o)}\left(z, x, x^{\prime}\right)=\frac{i}{2 z^{1 / 2}} \cdot e^{i z^{1 / 2}\left|x-x^{\prime}\right|}, \operatorname{Im}\left(z^{1 / 2}\right) \geq 0$ yields

$$
\left[\left(\beta+\partial_{1}\right)\left(\beta+\partial_{2}\right) G^{(o)}(z, x, x)\right]=(i / 2)\left[\beta^{2} z^{-1 / 2}+z^{1 / 2}\right], \quad \beta \in \mathrm{R}
$$

and

$$
\begin{gathered}
\xi_{\beta}^{(o)}(\lambda, x)= \begin{cases}0, & \lambda<-\beta^{2} \\
-1, & -\beta^{2}<\lambda<0, \quad \beta \in \mathrm{R} \backslash\{0\}, \\
-\frac{1}{2}, & \lambda>0\end{cases} \\
\xi_{o}^{(o)}(\lambda, x)= \begin{cases}0, & \lambda<0 \\
-\frac{1}{2}, & \lambda>0 .\end{cases}
\end{gathered}
$$

Thus

$$
\begin{gathered}
\operatorname{Tr}\left[\left(H_{\beta ; x}^{(o)}-z\right)^{-1}-\left(H^{(o)}-z\right)^{-1}\right]=\frac{\beta^{2}-z}{2 z\left(z+\beta^{2}\right)}, \quad \beta \in \mathrm{R}, z \in \mathrm{C} \backslash\left\{\left\{-\beta^{2}\right\} \cup[0, \infty)\right\}, \\
\operatorname{Tr}\left[e^{-t H_{\beta ; x}^{(o)}}-e^{-t H^{(o)}}\right]=-\frac{1}{2}+e^{t \beta^{2}}, \quad \beta \in \mathrm{R}, t>0
\end{gathered}
$$

where $H^{(o)}=-\frac{d^{2}}{d x^{2}}, \mathcal{D}\left(H^{(o)}\right)=H^{2,2}(\mathrm{R})$. One has

$$
\operatorname{spec}\left(H_{\beta ; x}^{(o)}\right)=\left\{-\beta^{2}\right\} \cup[0, \infty), \quad \beta \in \mathrm{R} .
$$

Next we recall the well-known fact that the Weyl $m$-functions $\phi_{ \pm}(z, x)$ associated with $H_{D, \pm ; x}$ in $L^{2}((x, \pm \infty)$ ) (see the paragraph following (5.23)) have the asymptotic expansion (5.18) as $z \rightarrow i \infty$ whenever $V$ satisfies (6.2), see [3], [23], [24]. (Actually the l.p. property of $h$ at $\pm \infty$ is irrelevant in this context and the asymptotic expansion (5.18) is valid outside any cone $|\tan \theta|<\epsilon$ for $\epsilon>0$ arbitrarily small.) Hence (5.23), (5.31), and (6.14) 
imply the existence of asymptotic expansions for $G(z, x, x), \frac{d}{d x} G(z, x, x), H(z, x, x)=$ $\left[\partial_{1} \partial_{2} G(z, x, x)\right]$, and $\frac{d}{d x} H(z, x, x)$ as $z \rightarrow i \infty$ to all orders in $z$. In the following we derive recursion relations for the coefficients in the expansion for $\left[\left(\beta+\partial_{1}\right)\left(\beta+\partial_{2}\right) G(z, x, x)\right]$ by reducing it to those of $G(z, x, x)$ and $H(z, x, x)$ under the assumptions (6.2) on $V$. The ansatz

$$
G(z, x, x) \underset{z \rightarrow i \infty}{\sim} \frac{i}{2} \sum_{j=0}^{\infty} g_{j}(x) z^{-j-1 / 2}
$$

inserted into the well-known differential equation for $G(z, x, x)$ (essentially equivalent to $(5.19))$

$$
4[V(x)-z] G(z, x, x)^{2}+\left[\frac{d}{d x} G(z, x, x)\right]^{2}-2 G(z, x, x)\left[\frac{d^{2}}{d x^{2}} G(z, x, x)\right]=1
$$

then yields the recursion relation $[10]$

$$
\begin{gathered}
g_{0}(x)=1, \quad g_{1}(x)=\frac{1}{2} V(x), \\
g_{j+1}(x)=-\frac{1}{2} \sum_{\ell=1}^{j} g_{\ell}(x) g_{j+1-\ell}(x)+\frac{1}{2} V(x) \sum_{\ell=0}^{j} g_{\ell}(x) g_{j-\ell}(x) \\
+\frac{1}{8} \sum_{\ell=0}^{j} g_{\ell}^{\prime}(x) g_{j-\ell}^{\prime}(x)-\frac{1}{4} \sum_{\ell=0}^{j} g_{\ell}^{\prime \prime}(x) g_{j-\ell}(x), \quad j \in \mathrm{N} .
\end{gathered}
$$

Equivalently, one could have used the linear third order equation

$$
\left[\frac{d^{3}}{d x^{3}} G(z, x, x)\right]-4[V(x)-z]\left[\frac{d}{d x} G(z, x, x)\right]+V^{\prime}(x) G(z, x, x)=0
$$

to obtain

$$
\begin{aligned}
& g_{0}(x)=1, \\
& g_{j}^{\prime}(x)=-\frac{1}{4} g_{j-1}^{\prime \prime \prime}(x)+V(x) g_{j-1}^{\prime}(x)+\frac{1}{2} V^{\prime}(x) g_{j-1}(x), \quad j \in \mathrm{N}
\end{aligned}
$$

which yields $g_{j}(x)$ upon (homogeneous) integration. Here $g_{j}$ are homogeneous differential polynomials in $V$ of degree

$$
\operatorname{deg}\left(g_{j}\right)=2 j, \quad j \in \mathrm{N}_{0}
$$

assuming $\operatorname{deg}\left(V^{(m)}\right)=m+2, m \in \mathrm{N}_{0}$. Explicitly, one obtains

$$
\begin{aligned}
g_{0}=1, \quad g_{1}(x)=\frac{1}{2} V(x), \quad g_{2}(x) & =\frac{3}{8} V(x)^{2}-\frac{1}{8} V^{\prime \prime}(x) \\
g_{3}(x) & =\frac{1}{32} V^{\prime \prime \prime \prime}(x)-\frac{5}{16} V(x) V^{\prime \prime}(x)-\frac{5}{32} V^{\prime}(x)^{2}+\frac{5}{16} V(x)^{3}, \quad \text { etc. }
\end{aligned}
$$


Equation (6.14) then yields

$$
\frac{d}{d x} H(z, x, x) \underset{z \rightarrow i \infty}{\sim} \frac{i}{2} \sum_{j=0}^{\infty}\left[V(x) g_{j}^{\prime}(x)-g_{j+1}^{\prime}(x)\right] z^{-j-1 / 2}
$$

and hence

$$
H(z, x, x) \underset{z \rightarrow i \infty}{\sim} \frac{i}{2} \sum_{j=0}^{\infty}\left[\int^{x} d x^{\prime} V\left(x^{\prime}\right) g_{j}^{\prime}\left(x^{\prime}\right)-g_{j+1}(x)\right] z^{-j-1 / 2}+C(z) .
$$

Here $\int^{x} d x^{\prime} V\left(x^{\prime}\right) g_{j}^{\prime}\left(x^{\prime}\right)$ denotes homogeneous integration, that is, all integration constants are put zero. Moreover, as proven in [10],

$$
g_{\ell}(x) g_{j}^{\prime}(x)=\frac{d}{d x} h_{\ell, j}(x)
$$

for some homogeneous differential polynomial $h_{\ell, j}$ in $V$ and hence $V g_{j}^{\prime}=2 g_{1} g_{j}^{\prime}$ is a total derivative (see (6.39)). The $x$-independent constant $C(z)$ in $(6.39)$ can be obtained from the free case $V(x) \equiv 0$ and one gets (cf. (6.18), (6.19))

$$
C(z)=i z^{1 / 2} / 2 \text {. }
$$

Alternatively, one could have used

$$
H(z, x, x)^{-1}=\phi_{+}(z, x)^{-1}-\phi(z, x)^{-1}
$$

and the asymptotic expansions (5.18) for $\phi_{ \pm}(z, x)$. Combining (6.12), (6.31), (6.39), and (6.41) then yields

$$
\begin{aligned}
{\left[\left(\beta+\partial_{1}\right)\left(\beta+\partial_{2}\right) G(z, x, x)\right]=\left(i z^{1 / 2} / 2\right)+(i / 2) \sum_{j=0}^{\infty}\left[\beta^{2} g_{j}(x)+\beta g_{j}^{\prime}(x)\right.} \\
\left.+\int^{x} d x^{\prime} V\left(x^{\prime}\right) g_{j}^{\prime}\left(x^{\prime}\right)-g_{j+1}(x)\right] z^{-j-1 / 2}=\left(i z^{1 / 2} / 2\right) \sum_{j=0}^{\infty} c_{\beta, j}(x) z^{-j}
\end{aligned}
$$

where

$$
\begin{aligned}
& c_{\beta, 0}(x)=1, \\
& c_{\beta, j}(x)=\beta^{2} g_{j-1}(x)+\beta g_{j-1}^{\prime}(x)+\int^{x} d x^{\prime} V\left(x^{\prime}\right) g_{j-1}^{\prime}\left(x^{\prime}\right)-g_{j}(x), \quad j \in \mathrm{N} .
\end{aligned}
$$

Explicitly, one gets

$$
\begin{gathered}
c_{\beta, 0}(x)=1, \quad c_{\beta, 1}(x)=\beta^{2}-\frac{1}{2} V(x), \\
c_{\beta, 2}(x)=\frac{1}{2} \beta^{2} V(x)+\frac{1}{2} \beta V^{\prime}(x)-\frac{1}{8} V(x)^{2}+\frac{1}{8} V^{\prime \prime}(x), \quad \text { etc. }
\end{gathered}
$$


Hence, applying (5.27)-(5.29) again, one infers

$$
\ln \left[\left(\beta+\partial_{1}\right)\left(\beta+\partial_{2}\right) G(z, x, x)\right]=\ln \left(i z^{1 / 2} / 2\right)+\sum_{j=1}^{\infty} d_{\beta, j}(x) z^{-j}
$$

where

$$
\begin{aligned}
& d_{\beta, 1}(x)=c_{\beta, 1}(x)=\beta^{2}-\frac{1}{2} V(x), \\
& d_{\beta, j}(x)=c_{\beta, j}(x)-\frac{1}{j} \sum_{\ell=1}^{j-1} \ell c_{\beta, j-\ell}(x) d_{\beta, \ell}(x), \quad j \geq 2 .
\end{aligned}
$$

Explicitly,

$$
\begin{aligned}
& d_{\beta, 1}(x)=\beta^{2}-\frac{1}{2} V(x), \\
& d_{\beta, 2}(x)=-\frac{1}{2} \beta^{4}+\beta^{2} V(x)+\frac{\beta}{2} V^{\prime}(x)-\frac{1}{4} V(x)^{2}+\frac{1}{8} V^{\prime \prime}(x), \quad \text { etc. }
\end{aligned}
$$

This finally leads to the following theorem.

Theorem 6.2. Suppose $V \in C^{\infty}(\mathrm{R}), V$ real-valued and bounded from below. Then for each $N \in \mathrm{N}$,

$$
\begin{aligned}
\operatorname{Tr}\left[\left(H_{\beta ; x}-z\right)^{-1}-(H-z)^{-1}\right] & =-\frac{d}{d z} \ln \left[\left(\beta+\partial_{1}\right)\left(\beta+\partial_{2}\right) G(z, x, x)\right] \\
& \sim \underset{z \rightarrow i \infty}{\sim} \sum_{j=0}^{N} r_{\beta, j}(z) z^{-j-1}+O\left(z^{-N-1}\right), \quad \beta \in \mathrm{R}, x \in \mathrm{R},
\end{aligned}
$$

where

$$
\begin{aligned}
& r_{\beta, 0}(x)=-\frac{1}{2}, \\
& r_{\beta, j}(x)=j c_{\beta, j}(x)-\sum_{\ell=1}^{j-1} c_{\beta, j-\ell}(x) r_{\beta, \ell}(x), \quad j \in \mathrm{N}
\end{aligned}
$$

with $c_{\beta, j}(x)$ computed from (6.44).

Proof. It suffices to note that

$$
r_{\beta, 0}(x)=-\frac{1}{2}, \quad r_{\beta, j}(x)=j d_{\beta, j}(x), \quad j \in \mathrm{N}
$$

upon differentiating (6.46) with respect to $z$.

Explicitly, one obtains from (6.50), (6.44),

$$
\begin{aligned}
& r_{\beta, 0}(x)=-\frac{1}{2}, \quad r_{\beta, 1}(x)=\beta^{2}-\frac{1}{2} V(x), \\
& r_{\beta, 2}(x)=-\beta^{4}+2 \beta^{2} V(x)+\beta V^{\prime}(x)-\frac{1}{2} V(x)^{2}+\frac{1}{4} V^{\prime \prime}(x), \quad \text { etc. }
\end{aligned}
$$

It remains to express $r_{\beta, j}(x)$ in terms of $\xi_{\beta}(\lambda, x)$ in analogy to the resolvent regularization procedure sketched in Remark 5.6. By exactly the same procedure one proves the following result. 
Theorem 6.3. Suppose $V \in C^{\infty}(\mathrm{R}), V$ real-valued and bounded from below. Assume that (4.2) holds and denote $E_{\beta, o}(x)=\inf \operatorname{spec}\left(H_{\beta ; x}\right)$. Then

$$
\begin{aligned}
r_{\beta, 0}(x) & =-\frac{1}{2}, \\
r_{\beta, 1}(x) & =\beta^{2}-\frac{1}{2} V(x) \\
& =\frac{E_{\beta, o}(x)}{2}+\lim _{z \rightarrow i \infty} \int_{E_{\beta, o}(x)}^{\infty} d \lambda \frac{z^{2}}{(\lambda-z)^{2}}\left[\frac{1}{2}-\xi(\lambda, x)\right], \\
r_{\beta, j}(x) & =\frac{E_{\beta, o}(x)^{j}}{2}+\lim _{z \rightarrow i \infty} \int_{E_{\beta, o}(x)}^{\infty} d \lambda \frac{z^{j+1}}{(\lambda-z)^{j+1}} j(-\lambda)^{j-1}\left[\frac{1}{2}-\xi(\lambda, x)\right], \quad j \in \mathrm{N}, x \in \mathrm{R} .
\end{aligned}
$$

Finally, the analog of Example 5.5 in the case where $V(x)$ is periodic reads as follows.

Example 6.4. Assume $V \in C^{\infty}(\mathrm{R}), V$ real-valued, for some $a>0, V(x+a)=V(x)$ for all $x \in \mathrm{R}$. Then the spectrum of $H$ is given by (5.55) while the spectrum of $H_{\beta ; x}$ is of the type

$$
\begin{aligned}
& \operatorname{spec}\left(H_{\beta ; x}\right)=\left\{\lambda_{\beta, n}(x)\right\}_{n \in \mathrm{N}_{0}} \cup \bigcup_{n=1}^{\infty}\left[E_{2(n-1)}, E_{2 n-1}\right], \\
& \lambda_{\beta, 0}(x) \leq E_{0}, \quad E_{2 n-1} \leq \lambda_{\beta, n}(x) \leq E_{2 n}, \quad n \in \mathrm{N} .
\end{aligned}
$$

The analog of (5.56) then reads

$$
\xi_{\beta}(\lambda, x)= \begin{cases}0, & \lambda<\lambda_{\beta, 0}(x), \quad E_{2 n-1}<\lambda<\lambda_{\beta, n}(x), \quad n \in \mathrm{N} \\ -1, & \lambda_{\beta, 0}(x)<\lambda<E_{0}, \quad \lambda_{\beta, n}(x)<\lambda<E_{2 n}, \quad n \in \mathrm{N} \\ -\frac{1}{2}, & E_{2(n-1)}<\lambda<E_{2 n-1}, \quad n \in \mathrm{N}\end{cases}
$$

and one obtains from (6.55) the higher order periodic trace formulas

$$
2 r_{\beta, j}(x)=E_{0}^{j}-2 \lambda_{\beta, 0}(x)^{j}+\sum_{n=1}^{\infty}\left[E_{2 n-1}^{j}+E_{2 n}^{j}-2 \lambda_{\beta, n}(x)^{j}\right], \quad \beta \in \mathrm{R}, j \in \mathrm{N}, x \in \mathrm{R} .
$$

While the periodic trace formulas (6.58) for $r_{\beta, j}(x)$ were known in the Neumann case $\beta=0$ [36] (see also [31]), the cases $\beta \in \mathrm{R} \backslash\{0\}$ in (6.58) appear to be new.

Acknowledgments. F.G. is indebted to the Department of Mathematical Sciences of the University of Trondheim, Norway, and the Department of Mathematics at Caltech, Pasadena, CA for the hospitality extended to him in the summer of 1993. Support by the Norwegian Research Council for Science and the Humanities (NAVF) (F.G. and H.H.) and by Caltech (F.G.) is gratefully acknowledged. 


\section{REFERENCES}

[1] N. Aronszajn and W.F. Donoghue, On exponential representations of analytic functions in the upper half-plane with positive imaginary part, J. Anal. Math. 5 (1957), 321-388.

[2] W. Craig, The trace formula for Schrödinger operators on the line, Commun. Math. Phys. 126 (1989), 379-407.

[3] A.A. Danielyan and B.M. Levitan, On the asymptotic behavior of the Weyl-Titchmarsh m-function, Math. USSR Izv. 36 (1991), 487-496.

[4] P. Deift and B. Simon, Almost periodic Schrödinger operators, III. The absolutely continuous spectrum in one dimension, Commun. Math. Phys. 90 (1983), 389-411.

[5] P. Deift and E. Trubowitz, Inverse scattering on the line, Commun. Pure Appl. Math. 32 (1979), 121-251.

[6] L.A. Dikii, Trace formulas for Sturm-Liouville differential operators, Amer. Math. Soc. Trans. Ser. (2) 18 (1961), 81-115.

[7] B.A. Dubrovin, Periodic problems for the Korteweg-de Vries equation in the class of finite band potentials, Funct. Anal. Appl. 9 (1975), 215-223.

[8] H. Flaschka, On the inverse problem for Hill's operator, Arch. Rat. Mech. Anal. 59 (1975), 293-309.

[9] I.M. Gel'fand, On identities for eigenvalues of a second order differential operator, Usp. Mat. Nauk 11:1 (1956), 191-198 (Russian); English transl. in Izrail M. Gelfand, Collected Papers Vol. I (S.G. Gindikin, V.W. Guillemin, A.A. Kirillov, B. Kostant, S. Sternberg, eds.) Springer-Berlin, 1987, pp. 510-517.

[10] I.M. Gel'fand and L.A. Dikii, Asymptotic behaviour of the resolvent of Sturm-Liouville equations and the algebra of the Korteweg-de Vries equations, Russ. Math. Surv. 30:5 (1975), 77-113.

[11] I.M. Gel'fand and B.M. Levitan, On a simple identity for eigenvalues of a second order differential operator, Dokl. Akad. Nauk SSSR 88 (1953), 593-596 (Russian); English transl. in Izrail M. Gelfand, Collected Papers Vol. I (S.G. Gindikin, V.W. Guillemin, A.A. Kirillov, B. Kostant, S. Sternberg, eds.) Springer, Berlin, 1987, pp. 457-461.

[12] F. Gesztesy, New trace formulas for Schrödinger operators, Evolution Equations (G. Ferreyra, G. Goldstein, F. Neubrander, eds.), Marcel Dekker, to appear.

[13] F. Gesztesy and H. Holden, Trace formulas and conservation laws for nonlinear evolution equations, Rev. Math. Phys., (to appear).

[14] F. Gesztesy, H. Holden, and B. Simon, Absolute summability of the trace relation for certain Schrödinger operators, to be submitted to Commun. Math. Phys.

[15] F. Gesztesy, H. Holden, B. Simon, and Z. Zhao, Trace formulae and inverse spectral theory for Schrödinger operators, Bull. Amer. Math. Soc. 29 (1993), 250-255.

[16] F. Gesztesy, H. Holden, B. Simon, and Z. Zhao, A trace formula for multidimensional Schrödinger operators, to be submitted to Invent. Math.

[17] F. Gesztesy and B. Simon, Rank one perturbations at infinite coupling, to be submitted to J. Funct. Anal.

[18] F. Gesztesy and B. Simon, The xi function, to be submitted to Ann. Math.

[19] F. Gesztesy and B. Simon, Inverse spectral theory for one-dimensional Schrödinger operators, in preparation. 
[20] F. Gesztesy and B. Simon, Inverse spectral theory for one-dimensional Jacobi matrices, in preparation.

[21] R.C. Gilbert and V.A. Kramer, Trace formulas for powers of a Sturm-Liouville operator, Canad. J. Math. 16 (1964), 412-422.

[22] C.J.A. Halberg and V.A. Kramer, A generalization of the trace concept, Duke Math. J. 27 (1960), 607-617.

[23] B.J. Harris, On the Titchmarsh-Weyl m-function, Proc. Roy. Soc. Edinburgh 95A (1983), 223-237.

[24] B.J. Harris, The asymptotic form of the Titchmarsch-Weyl m-function, J. London Math. Soc. (2), 30 (1984), 110-118.

[25] H. Hochstadt, On the determination of a Hill's equation from its spectrum, Arch. Rat. Mech. Anal. 19 (1965), 353-362.

[26] K. Iwasaki, Inverse problem for Sturm-Liouville and Hill equation, Ann. Mat. Pura Appl. Ser. 4, 149 (1987), 185-206.

[27] R. Johnson and J. Moser, The rotation number for almost periodic potentials, Commun. Math. Phys. 84 (1982), 403-438.

[28] S. Kotani, Ljapunov indices determine absolutely continuous spectra of stationary random one-dimensional Schrödinger operators, Stochastic Analysis (K. Ito, ed.), NorthHolland, Amsterdam, 1984, pp. 225-247.

[29] S. Kotani and M. Krishna, Almost periodicity of some random potentials, J. Funct. Anal. 78 (1988), 390-405.

[30] M. G. Krein, Perturbation determinants and a formula for the traces of unitary and self-adjoint operators, Sov. Math. Dokl. 3 (1962), 707-710.

[31] P.D. Lax, Trace formulas for the Schrödinger operator, Commun. Pure Appl. Math., to appear.

[32] B.M. Levitan, On the closure of the set of finite-zone potentials, Math. USSR Sbornik 51 (1985), 67-89.

[33] B.M. Levitan, Inverse Sturm-Liouville Problems, VNU Science Press, Utrecht, 1987.

[34] V.A. Marchenko, Sturm-Liouville Operators and Applications, Birkhäuser, Basel, 1986.

[35] H.P. McKean and P. van Moerbeke, The spectrum of Hill's equation, Invent. Math. 30 (1975), 217-274.

[36] H.P. McKean and E. Trubowitz, Hill's operator and hyperelliptic function theory in the presence of infinitely many branch points, Commun. Pure Appl. Math. 29 (1976), $143-226$.

[37] B. Simon, Functional Integration and Quantum Physics, Academic Press, New York, 1979.

[38] B. Simon, Schrödinger semigroups, Bull. Amer. Math. Soc. 7 (1982), 447-526.

[39] B. Simon, Spectral analysis of rank one perturbations and applications, Lecture given at the 1993 Vancouver Summer School, preprint.

[40] E. Trubowitz, The inverse problem for periodic potentials, Commun. Pure Appl. Math. 30 (1977), 321-337.

[41] S. Venakides, The infinite period limit of the inverse formalism for periodic potentials, Commun. Pure Appl. Math. 41 (1988), 3-17. 